\title{
IV. INFLUENCE DE LA CONDUITE DU SÉCHAGE A LA FLAMME SUR L'EFFICACITÉ DES PROTÉINES DE FARINES DE HARENG
}

\author{
I N T R O D U C T I O N \\ S. Z. ZELTER \\ Laboratoire de Recherches sur la Conservation et l'Efficacité des Aliments, \\ 16 , rue Claude Bernard, Paris $V^{\mathrm{e}}$.
}

L'intérêt des protéines animales, fabriquées industriellement, en alimentation animale n'est plus à démontrer. Le résultat zootechnique est essentiellement contingenté par leur qualité. Parmi les facteurs susceptibles d'influencer celle-ci, la conduite du traitement thermique est prépondérante en raison de la labilité particulière de certains acides aminés essentiels.

L'influence de l'intensité du chauffage sur l'efficacité protidique des farines de poisson a été mise en évidence par des travaux anciens, passés en revue par Creach (1950) Grau et Williams (1955) Ousterhout et Snyder (1962) et d'après lesquels le séchage à la flamme (WILgus 1935) serait particulièrement préjudiciable. Selon des études plus récentes (TARR et al., 1954, CARPENTER et al., 1954) les progrès de la technologie permettent d'obtenir par ce procédé des produits de qualité comparable à celle de farines séchées sous vide ou à la vapeur. On peut néanmoins se demander si dans cette technique certaines zones thermiques ne seraient pas plus favorables que d'autres à la protection des protéines de poisson. C'est ce point qui a fait l'objet de recherches exposées plus loin. Mais le mode de séchage n'est pas le seul à influencer l'efficacité azotée d'une farine animale; d'autres variables peuvent également la modifier : origine et composition de la matière première, durée et température de la cuisson précédant le séchage, échauffement spontané des farines à la sortie du séchoir, conditions de stockage. Une interprétation valable des résultats expérimentaux impliquait donc nécessairement l'élimination préalable de l'interférence de tous les facteurs autres que la conduite du séchage. 
Dans ce but, trois farines ont été préparées à partir d'un même lot de pêche de hareng d'hiver par voie humide et sans réincorporation de la fraction soluble séparée au cours du pressage. Ces trois produits fabriqués dans l'usine pilote de l'Institut de Recherches sur les huiles et farines de poisson de TJakrevick (Norvège), se distinguent entre eux uniquement par les conditions de leur séchage à la flamme. Ces conditions sont résumées dans la tableau 1.

\section{TABleau 1}

Conditions de séchage des farines de hareng.

\begin{tabular}{|c|c|c|c|}
\hline & $\begin{array}{c}\text { Farine } \\
\text { de hareng } \\
60^{\circ}\end{array}$ & $\begin{array}{c}\text { Farine } \\
\text { de hareng } \\
90^{\circ}\end{array}$ & $\begin{array}{c}\text { Farine } \\
\text { de hareng } \\
130^{\circ}\end{array}$ \\
\hline Température des gaz à l'admission ........... & $490-520^{\circ} \mathrm{C}$ & $750^{\circ} \mathrm{C}$ & $600^{\circ} \mathrm{C}$ \\
\hline 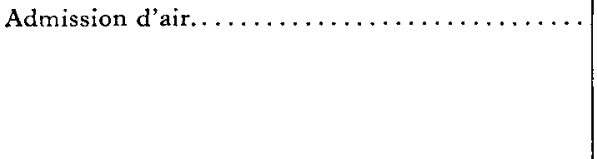 & $\begin{array}{l}\text { limitée en vue } \\
\text { de la juste } \\
\text { évacuation d'eau }\end{array}$ & limitée & $\begin{array}{l}\text { Excès; chaleur } \\
\text { fournie supé- } \\
\text { rieure à celle } \\
\text { nécessaire pour } \\
\text { éliminer l'eau }\end{array}$ \\
\hline Sortie du four : & & & \\
\hline - Atmosphère...$\ldots \ldots \ldots \ldots \ldots \ldots$ & saturée d'eau & 一 & non saturée d'eau \\
\hline - Température...................... & $60^{\circ} \mathrm{C}$ & $90-95^{\circ} \mathrm{C}$ & $130^{\circ} \mathrm{C}$ \\
\hline Observations $\ldots \ldots \ldots \ldots \ldots \ldots \ldots \ldots \ldots \ldots \ldots \ldots$ & $\begin{array}{c}\text { Séchage } \\
\text { économique }\end{array}$ & $\begin{array}{l}\text { Traversée lente } \\
\text { du four, risque } \\
\text { de combustion }\end{array}$ & $\begin{array}{c}\text { Traversée rapide } \\
\text { du four }\end{array}$ \\
\hline
\end{tabular}

Les trois échantillons sont désignés par des nombres qui correspondent approximativement aux températures, à la sortie du four; mais qui ne s'accordent pas directement avec les températures des gaz à l'entrée; en effet, la quantité d'air secondaire admise, facteur qui détermine le temps de transit de la masse et donc la durée de chauffage, n'a malheureusement pas pu être déterminée d'une manière précise.

A leur arrivée en France, les farines de hareng ont été conservées pendant un an à $-15^{\circ} \mathrm{C}$, avant d'être expérimentées.

L'étude effectuée constitue une véritable œuvre coopérative de plusieurs laboratoires (Centre de Recherches sur la Nutrition du C.N.R.S., Station de Recherches Avicoles, Laboratoire des Métabolismes, et Station de Recherches sur l'élevage des Porcs du C.N.R.Z., Laboratoire de Recherches sur la Conservation et l'Efficacité des Aliments de l'I.N.R.A.); elle est un exemple typique d'une recherche concertée en nutrition animale. Son intérêt réside dans le fait qu'elle a été réalisée sur trois espèces animales : rat, poulet et porc. Les besoins spécifiques en acides aminés n’étant pas identiques pour ces trois espèces, il était curieux d'observer leur réaction à un même traitement thermique.

En l'occurrence, il est remarquable de noter que les techniques expérimentales mises en œuvre diffèrent avec le laboratoire et le réactif animal. Les unes sont basées 
sur des bilans d'azote (rétention et V. B.) qui mettent plus particulièrement en lumière la déficience d'un aliment constituant la source exclusive d'un acide aminé déterminé qui aurait pu être rendu indisponible par un traitement thermique inadéquat et devenir alors le facteur limitant primaire; c'est particulièrement le cas des acides aminés soufrés dont le taux est limité pour la farine de poisson. Les autres sont des tests de croissance qui permettent plus spécialement d'estimer la valeur de supplémentation d'un aliment en un acide aminé essentiel tel la lysine pour corriger le déficit d'un régime en ce facteur limitant primaire, dont la sensibilité thermique est très grande.

Les résultats expérimentaux rapportés ici montrent que quels que soient la méthode expérimentale et le réactif animal employés, les réponses sont assez concordantes : aucune des trois zones thermiques étudiées dans le cadre du procédé de séchage à la flamme, ne paraît supérieure aux deux autres pour ce qui concerne le degré de protection de la disponibilité des acides aminés soufrés et de la lysine.

Les légers écarts enregistrés dans certains laboratoires entre les échantillons pourraient être imputés à des différences dans la durée de stockage, en raison du décalage des essais dans le temps.

\section{REFERENCES BIBLIOGRAPHIQUES}

Carpenter G., Ellinger M., Shrimpton D.H., 1954 - The nutritive value of six white fish meals of known origin. Froc. Nutri. Soc., 13, 20-21.

Creac' H P. V., 1950 - Congrès International d'études sur le rôle du poisson dans l'alimentation, 249-294, Institut Océanographique, Paris.

Grau C. R., Williams M. A., 1955 - Fish meals as amino-acid sources in chick rations. Poultry Sci., 34, 810-817.

Ousterhout L. E., Snyder D. G., 1962, in Heen E., Kreuzer R. - Fish in nutrition, 303-309, Fishing news (books) Ltd, London.

Tarr H. L. A., Biely J., March B. E., 1954 - The nutritive value of herring meals. 1. The effect of heat. Poultry Sci., 33, 242-250.

Wilgus H.S. Jr, Norris L. C., Heuser G. F., 1935 - Haddock meal. Effect of manufacturing process upon nutritive value. Indust. Eng. Chem., 27, 419-422. 